\title{
A Note on Asymptotic Equilibrium for Fuzzy Differential Equations
}

\author{
Yabin Shao, ${ }^{1,2}$ Shengui Zhang, ${ }^{2}$ and Yanping Xiao ${ }^{2}$ \\ ${ }^{1}$ Department of Mathematics, Sichuan University, Chengdu 610065, China \\ ${ }^{2}$ College of Mathematics and Computer Sciences, Northwest University for Nationalities, Lanzhou 730030, China \\ Correspondence should be addressed to Yabin Shao; yb-shao@163.com
}

Received 26 November 2013; Accepted 23 January 2014; Published 5 March 2014

Academic Editor: Junjie Wei

Copyright (c) 2014 Yabin Shao et al. This is an open access article distributed under the Creative Commons Attribution License, which permits unrestricted use, distribution, and reproduction in any medium, provided the original work is properly cited.

\begin{abstract}
The asymptotic equilibrium results for fuzzy differential systems $(G C P) x^{\prime}=f_{1}(t, x, y), y^{\prime}=f_{2}(t, x, y)$ are investigated, where $f_{1}(t, x, y)$ satisfies the compactness-type and $f_{2}(t, x, y)$ satisfies the dissipative-type conditions. It is worth mentioning that the uniformly continuous conditions of $f(t, x, y)$ are removed in Song et al. (2005). That is to say, the results of Song et al. (2005) are extended. In addition, the global existence and asymptotic equilibrium results of fuzzy differential systems $(C P) x^{\prime}(t)=$ $f(t, x), x(0)=x_{0}$ are obtained.
\end{abstract}

\section{Introduction}

The Cauchy problems for fuzzy differential equations have been studied by several authors [1-6] on the metric space $\left(E^{n}, D\right)$ of normal fuzzy convex set with the distance $D$ given by the maximum of the Hausdorff distance between the corresponding level sets. In [4], Nieto proved the Cauchy problems which have a unique solution if $f$ is continuous and bounded. For a general reference to fuzzy differential equations, see a recent book by Lakshmikantham and Mohapatra [7] and references therein. In particular, Wu and Song [8-10] changed the initial value problem of fuzzy differential equations into abstract differential equations on a closed convex cone in a Banach space by the operator $j$ that is the isometric embedding from $\left(E^{n}, D\right)$ onto its range in the Banach space $X$. They established the relationships between a solution and its approximate solutions to fuzzy differential equations. Furthermore, they obtained the local existence theorems under the compactness-type and the dissipativetype conditions. Park and Han [11] showed the global existence and uniqueness of fuzzy solutions of fuzzy differential equation using the properties of Hasegawa's function and successive approximation. Song et al. [12] pointed out a variety of results which assure global existence of solutions to fuzzy differential equations. Song et al. [13] studied the asymptotic equilibrium for fuzzy differential equations:

$$
x^{\prime}=f(t, x), \quad x\left(t_{0}\right)=x_{0},
$$

where $x_{0} \in E^{n}$ and $f$ is a continuous. Since we are only interested in local solutions to $(C P)$, we assume $f: J \times B \rightarrow$ $E^{n}$ and $D(f, \widetilde{0}) \leq M$ on $J \times B$ for some $M$, where $J=[0, a]$ and $B=\bar{B}\left(x_{0}, r\right)=\left\{x \in E^{n}: D\left(x, x_{0}\right) \leq r\right\}$. The methods used by them are as follows: $f$ satisfied not only the compactness-type conditions but the uniformly continuity condition. Hence, it is of significance to lessen the growth conditions of $f$.

Based on those preceding works, in this paper, we firstly give the existence theorems for $(C P)$ without a uniform continuity assumption on $f$. In addition, the more general systems than $(C P)$ are considered:

$$
x^{\prime}=\left(\begin{array}{l}
x^{\prime} \\
y^{\prime}
\end{array}\right)=\left(\begin{array}{l}
f_{1}(t, x, y) \\
f_{2}(t, x, y)
\end{array}\right)=f(t, z)
$$

where $z=\left(\begin{array}{l}x \\ y\end{array}\right), f \in C\left[\left[t_{0},+\infty\right) \times E^{n}, E^{n}\right], f=\left(\begin{array}{l}f_{1} \\ f_{2}\end{array}\right)$, and $E_{1}^{n}$ and $E_{2}^{n}$ are the fuzzy number space, respectively. $f_{i} \in C\left[\left[t_{0},+\infty\right) \times E^{n}, E_{i}^{n}\right](i=1,2), f_{1}(t, x, y)$ satisfies 
the compactness-type, and $f_{2}(t, x, y)$ satisfies the dissipativetype conditions. In particular, when $E^{n}=E_{1}^{n}$ and $f_{2}=0$, we obtain that Corollary 24 is the promotion of the results of [13]. When $E^{n}=E_{2}^{n}$ and $f_{1}=0$, we obtain that Corollary 26 is the asymptotic equilibrium of fuzzy differential system $(C P)$ under the dissipative-type conditions.

As preliminaries we recall some basic results on fuzzy number space $\left(E^{n}, D\right)$ and list several comparison theorems on classical ordinary differential equations. In Section 3, we will proof the existence theorems for $(C P)$ without a uniform continuity assumption on $f$. In Section 4 , we will show that the asymptotic equilibrium for fuzzy differential system $(G C P)$. Finally, in Section 5, we present some concluding remarks.

\section{Preliminaries}

Let $P_{k}\left(R^{n}\right)$ denote the family of all nonempty compact convex subsets of $R^{n}$ and define the addition and scalar multiplication in $P_{k}\left(R^{n}\right)$ as usual. Let $A$ and $B$ be two nonempty bounded subsets of $R^{n}$. The distance between $A$ and $B$ is defined by the Hausdorff metric:

$$
d_{H}(A, B)=\max \left\{\sup _{a \in A} \inf _{b \in B}\|a-b\|, \sup _{b \in B} \inf _{a \in A}\|b-a\|\right\} .
$$

Denote $E^{n}=\left\{u: R^{n} \rightarrow[0,1] \mid u\right.$ satisfies (1)-(4) below $\}$ is a fuzzy number space, where

(1) $u$ is normal; that is, there exists an $x_{0} \in R^{n}$ such that $u\left(x_{0}\right)=1$;

(2) $u$ is fuzzy convex; that is, $u(\lambda x+(1-\lambda) y) \geq$ $\min \{u(x), u(y)\}$ for any $x, y \in R^{n}$ and $0 \leq \lambda \leq 1 ;$

(3) $u$ is upper semicontinuous;

(4) $[u]^{0}=\operatorname{cl}\left\{x \in R^{n} \mid u(x)>0\right\}$ is compact.

For $0<\alpha \leq 1$, denote $[u]^{\alpha}=\left\{x \in R^{n} \mid u(x) \geq \alpha\right\}$. Then from above (1)-(4), it follows that the $\alpha$-level set $[u]^{\alpha} \in$ $P_{k}\left(R^{n}\right)$ for all $0 \leq \alpha<1$. According to Zadeh's extension principle, we have addition and scalar multiplication in fuzzy number space $E^{n}$ as follows:

$$
[u+v]^{\alpha}=[u]^{\alpha}+[v]^{\alpha}, \quad[k u]^{\alpha}=k[u]^{\alpha},
$$

where $u, v \in E^{n}$ and $0 \leq \alpha \leq 1$.

Define $D: E^{n} \times E^{n} \rightarrow[0, \infty):$

$$
D(u, v)=\sup \left\{d_{H}\left([u]^{\alpha},[v]^{\alpha}\right): \alpha \in[0,1]\right\}
$$

where $d$ is the Hausdorff metric defined in $P_{k}\left(R^{n}\right)$. Then it is easy to see that $D$ is a metric in $E^{n}$. Using the results in $[14,15]$, we know that $\left(E^{n}, D\right)$ is a complete metric space. It is well known that application of fuzzy set theory very often involves the metric space $\left(E^{n}, D\right)$ of normal fuzzy convex set over $R^{n}$, where $D$ is the supremum of the Hausdoff distance between corresponding level sets. This metric has been found very convenient in studying of fuzzy differential equations (see [7]).
Definition 1 (see $[16,17]$ ). Let $S^{n-1}$ be the unit sphere of $R^{n}$; that is, $S^{n-1}=\{x \in R \mid\|x\|=1\},\langle\cdot, \cdot\rangle$ is the inner product in $R^{n}$; that is, $\langle x, y\rangle=\sum_{i=1}^{n} x_{i} y_{i}$, where $x=\left\langle x_{1}, x_{2}, \ldots, x_{n}\right\rangle$, $y=\left\langle y_{1}, y_{2}, \ldots, y_{n}\right\rangle \in R^{n}$. Suppose $u \in E^{n}, x \in S^{n-1}$, and $r \in$ $[0,1]$; then the support function of $u$ is defined by $u^{*}(r, x)=$ $\sup _{\alpha \in[u]^{\alpha}}\langle\alpha, x\rangle$ for all $(r, x) \in I \times S^{n-1}$.

The properties of the support functions can be referred to in [17] for details.

Theorem 2 (see $[16,17])$. Let $S^{n-1}$ be the unit sphere of $R^{n}$ and $\langle\cdot, \cdot\rangle$ the inner product in $R^{n}$. Suppose $u \in E^{n}, u^{*}(r, x)$ is the support function of $u$; then

(1) $u^{*}(r, x) \leq \sup _{a \in[u]^{r}}\|a\|$; that is, $u^{*}(r, x)$ is bounded on $S^{n-1}$, for each fixed $r \in[u]^{r}$;

(2) $u^{*}(r, x)$ is nonincreasing and left continuous in $r \epsilon$ $(0,1]$ and right continuous at $r=0$, for each fixed $x \in S^{n-1}$;

(3) $u^{*}(r, x)$ is Lipschitz continuous in $x$ and

$$
\left\|u^{*}(r, x)-u^{*}(r, y)\right\| \leq\left(\sup _{a \in[u]^{r}}\|a\|\right)\|x-y\| ;
$$

(4) if $u, v \in E^{n}, r \in[0,1]$, then $d\left([u]^{r},[v]^{r}\right)=\sup _{x \in S^{n-1}}$ $\left|u^{*}(r, x)-v^{*}(r, x)\right|$.

Theorem 3 (see $[16,17])$. Define an operator $j$ on $E^{n}$ as $j(u)(r, x)=u^{*}(r, x)$ for any $u \in E^{n}$; then one has

(1) $j(u) \in \bar{C}\left(I, C\left(S^{n-1}\right)\right)$,

(2) $j(s u+t v)=s j(u)+t j(v), s, t \geq 0$,

(3) $\|j(u)-j(v)\|=D(u, v)$,

(4) $j\left(E^{n}\right)$ is closed in $\bar{C}\left(I, C\left(S^{n-1}\right)\right)$.

In the sequel we will recall some integrability and differentiability properties in [18-20] for fuzzy set-valued mappings.

Let $T=\left[t_{0}, t_{0}+p\right] \subset R(p>0)$ be compact interval. The fuzzy mapping $F: T \rightarrow E^{n}$ is called strong measurable if for all $\alpha \in[0,1]$ the set-valued mapping $F_{\alpha}: T \rightarrow$ $P_{k}\left(R^{n}\right)$ defined by $F_{\alpha}(t)=[F(t)]^{\alpha}$ is Lebesgue measurable, where $P_{k}\left(R^{n}\right)$ is endowed with the topology generated by the Hausdorff metric $d$. A mapping $F_{\alpha}: T \rightarrow E^{n}$ is called integrable bounded if there exists an integrable function $h$ such that $\|x\| \leq h(t)$ for all $x \in F_{0}(t)$.

Definition 4. Let $F: T \rightarrow E^{n}$. The integral of $F$ over $T$, denoted by $\int_{T} F(t) d t$, is defined level-wise by the equation (see [18]):

$$
\begin{aligned}
{\left[\int_{T} F(t) d t\right]^{\alpha} } & =\int_{T} F_{\alpha}(t) d t \\
& =\left\{\int_{T} f(t) d t \mid f: T \rightarrow R^{n}\right.
\end{aligned}
$$

is a measurable selection for $\left.F_{\alpha}\right\}$. 
A strongly measurable and integrable mapping $F_{\alpha}: T \rightarrow$ $E^{n}$ is said to be integrable over $T$ if $\int_{T} F(t) d t \in E^{n}$. From [20], we know that if $F_{\alpha}: T \rightarrow E^{n}$ is continuous, then it is integrable.

Let $x, y \in E^{n}$. If there exists a fuzzy number $z \in E^{n}$ such that $x=y+z, z$ is called the $H$-difference of $x$ and $y$ that is denoted by $x-{ }_{H} y$. For brevity, we always assume that it satisfies the $H$-difference when dealing with the operation of subtraction of fuzzy numbers throughout this paper.

Definition 5 (see [20]). A mapping $F: T \rightarrow E^{n}$ is differentiable at $t_{0} \in T$ if there exists a $F^{\prime}\left(t_{0}\right) \in E^{n}$, such that the limits

$$
\lim _{h \rightarrow 0^{+}} \frac{F\left(t_{0}+h\right)-{ }_{H} F\left(t_{0}\right)}{h}, \quad \lim _{h \rightarrow 0^{+}} \frac{F\left(t_{0}\right)-{ }_{H} F\left(t_{0}+h\right)}{h}
$$

exist and are equal to $F^{\prime}\left(t_{0}\right)$.

Here the limits are taken in the metric space $\left(E^{n}, D\right)$. At the endpoint of $T$, we consider only one-side fuzzy derivatives. If $F: T \rightarrow E^{n}$ is differentiable at $t_{0} \in T$, then we say that $F^{\prime}\left(t_{0}\right)$ is the fuzzy derivative of $F(t)$ at the point $t_{0}$.

We note that this definition is fairly strong, because the family of fuzzy-number-valued functions $H$-differentiable is very restrictive. For example, the fuzzy-number-valued function $f:[a, b] \rightarrow R_{\mathscr{F}}$ defined by $f(x)=C \cdot g(x)$, where $C$ is a fuzzy number, $\cdot$ is the scalar multiplication (in the fuzzy context), and $g:[a, b] \rightarrow R^{+}$, with $g^{\prime}\left(t_{0}\right)<0$, is not $H$ differentiable in $t_{0}$ (see $\left.[19,21]\right)$. To avoid the above difficulty, in this paper we consider a more general definition of a derivative for fuzzy-number-valued functions enlarging the class of differentiable fuzzy-number-valued functions, which has been introduced in [19].

Definition 6 (see [19]). Let $f:(a, b) \rightarrow E^{n}$ and $x_{0} \in(a, b)$. One says that $f$ is differentiable at $x_{0}$ if there exists an element $f^{\prime}\left(t_{0}\right) \in E^{n}$, such that,

(1) for all $h>0$ sufficiently small, there exist $f\left(x_{0}+\right.$ $h){ }_{H} f\left(x_{0}\right), f\left(x_{0}\right){ }_{H} f\left(x_{0}-h\right)$ and the limits (in the metric D)

$$
\begin{aligned}
\lim _{h \rightarrow 0} \frac{f\left(x_{0}+h\right)-{ }_{H} f\left(x_{0}\right)}{h} \\
\quad=\lim _{h \rightarrow 0} \frac{f\left(x_{0}\right)-{ }_{H} f\left(x_{0}-h\right)}{h}=f^{\prime}\left(x_{0}\right)
\end{aligned}
$$

(2) for all $h>0$ sufficiently small, there exist $f\left(x_{0}\right){ }_{-}{ }_{H}$ $f\left(x_{0}+h\right), f\left(x_{0}-h\right){ }_{H} f\left(x_{0}\right)$ and the limits

$$
\begin{aligned}
\lim _{h \rightarrow 0} \frac{f\left(x_{0}\right)-{ }_{H} f\left(x_{0}+h\right)}{-h} \\
\quad=\lim _{h \rightarrow 0} \frac{f\left(x_{0}-h\right)-{ }_{H} f\left(x_{0}\right)}{-h}=f^{\prime}\left(x_{0}\right)
\end{aligned}
$$

(3) for all $h>0$ sufficiently small, there exist $f\left(x_{0}+\right.$ $h){ }_{H} f\left(x_{0}\right), f\left(x_{0}-h\right){ }_{H} f\left(x_{0}\right)$ and the limits

$$
\begin{aligned}
\lim _{h \rightarrow 0} & \frac{f\left(x_{0}+h\right)-{ }_{H} f\left(x_{0}\right)}{h} \\
\quad & \lim _{h \rightarrow 0} \frac{f\left(x_{0}-h\right)-{ }_{H} f\left(x_{0}\right)}{-h}=f^{\prime}\left(x_{0}\right)
\end{aligned}
$$

(4) for all $h>0$ sufficiently small, there exist $f\left(x_{0}\right)-{ }_{H}$ $f\left(x_{0}+h\right), f\left(x_{0}\right){ }_{-} f\left(x_{0}-h\right)$ and the limits

$$
\begin{aligned}
\lim _{h \rightarrow 0} \frac{f\left(x_{0}\right)-{ }_{H} f\left(x_{0}+h\right)}{-h} \\
\quad=\lim _{h \rightarrow 0} \frac{f\left(x_{0}\right)-{ }_{H} f\left(x_{0}-h\right)}{h}=f^{\prime}\left(x_{0}\right)
\end{aligned}
$$

( $h$ and $-h$ at denominators mean $1 / h$ and $-1 / h$, resp.).

In addition, we define a continuous fuzzy-valued function $f: T \times \Omega \rightarrow E^{n}$ by $f \in C\left[T \times \Omega, E^{n}\right]$, where $\Omega \subset E^{n}$ is an open set.

Theorem 7 (see [19]). Assume that $f \in C\left[T \times \Omega, E^{n}\right] . A$ function $x: T \rightarrow \Omega$ is a solution to the problem $x^{\prime}=f(t, x)$, $x\left(t_{0}\right)=x_{0}$ if and only if it is continuous and satisfies the integral equation

$$
x(t)=x_{0}+\int_{t_{0}}^{t} f(s, x(s)) d s
$$

or

$$
x(t)=x_{0}+(-1) \cdot \int_{t_{0}}^{t} f(s, x(s)) d s
$$

for all $t \in T$.

Theorem 8 (see [22]). Let $G \subset R^{2}$ be an open set, $g \in$ $C\left[G, R^{1}\right],\left(t_{0}, u_{0}\right) \in G$. Suppose that the maximum solution of initial value problem $u^{\prime}(t)=g(t, u), u\left(t_{0}\right)=u_{0}$ is $r(t)$ and its largest interval of existence of right solution is $\left[t_{0}, t_{0}+a\right)$. If $m(t) \in C\left[\left[t_{0}, t_{0}+a\right), R^{1}\right]$ satisfies $(t, m(t)) \in G$, for all $t \in\left[t_{0}, t_{0}+a\right), m\left(t_{0}\right) \leq u_{0}$ and

$$
D m(t) \leq g(t, m(t)), \quad \forall t \in\left[t_{0}, t_{0}+a\right) \backslash \Gamma,
$$

where $D$ is one of the four Dini derivatives and $\Gamma$ at most is a countable set on $\left[t_{0}, t_{0}+a\right)$. Then one has $m(t) \leq r(t)$, for all $t \in\left[t_{0}, t_{0}+a\right)$.

Theorem 9 (see [22], (Ascoli-Arzela)). A set $H \subset C(T, X)$ is a relative compact set if and only if $H$ is equicontinuous and, for any $t \in T, H(t)$ is relative compact set in $X$. 


\section{On the Cauchy Problem for Fuzzy Differential Equations}

Let $\gamma$ be Kuratowski's measure of noncompactness (see [22] for details).

Lemma 10. Let $S, T$ are two bounded subsets of Banach space $X$ and $t$ a real number; then Kuratowski's measure of noncompactness has following properties:

(1) $\gamma(S)=0$ if and only if $S$ is a relatively compactness set,

(2) $S \subset T \Rightarrow \gamma(S) \leq \gamma(T)$,

(3) $\gamma(t S)=|t| \gamma(S)$, where $t S=\{t x: x \in S\}$,

(4) $\gamma(S+T) \leq \gamma(S)+\gamma(T)$, where $S+T=\{x+y: x \in$ $S, y \in T\}$,

(5) $\gamma(S \times T)=\max \{\gamma(S), \gamma(T)\}$, where $S \times T$ is the bounded subsets of Banach space $E_{1} \times E_{2}$.

It is well known that continuity of $f$ is not sufficient for the existence of local solution to $(C P)$ (see [4]). The extra conditions that have been imposed on $f$ are mainly estimates which guarantee that a certain sequence of approximate solutions has at least a uniformly convergent subsequence. In this section, we would like to dispense with the uniform continuity assumption of $f$.

Next, we describe a class of uniqueness functions. Let $J \subset$ $R$ be an interval. A function $\omega: J \times R^{+} \rightarrow R$ is said to satisfy Caratheodory's condition on $J \times R^{+}$if $\omega(t, x)$ is measurable in $t$ for $x \in R^{+}$, continuous in $x$ for $t \in J$ and such that to each $x_{0}>0$ and compact interval $J_{0} \subset J$ there exists a function $h_{0} \in L^{1}\left(J_{0}\right)$ with $|\omega(t, x)| \leq h_{0}(t)$ in $J_{0} \times\left[0, x_{0}\right]$.

Definition 11 (see [23]). A function $\omega:(0, a] \times R^{+} \rightarrow R^{+}$with $\omega \in K\left((0, a] \times R^{+}\right)$is said to be class $U$, denoted by $\omega \in U$, if $\phi^{\prime}(t) \leq \omega(t, \phi(t))$ a.e. on $(0, a]$ and $\phi(t)=o(t)$ for $t \rightarrow$ $0^{+}$imply $\phi \equiv 0$ on $[0, a]$ for each absolutely continuous $\phi$ : $[0, a] \rightarrow R^{+}$.

We will consider a sequence of fuzzy-number-valued functions behaving like the sequence of approximate solutions that we will use in next section.

Theorem 12. Let $x_{n}$ be a sequence of continuous fuzzynumber-valued functions from $J=[a, b]$ to $E^{n}$ such that there is some function $\mu \in L^{1}(a, b)$ with $D\left(x_{n}(t), \widetilde{0}\right) \leq \mu(t)$ on J. Let $\phi(t)=\gamma\left(j \circ\left\{x_{n}(t): n \in N\right\}\right)$. Then $\phi(t)$ is integrable on $J$ and

$$
\gamma\left(j \circ\left\{\int_{a}^{b} x_{n}(\tau) d \tau: n \in N\right\}\right) \leq \int_{a}^{b} \phi(\tau) d \tau .
$$

Proof. Fix $n, m \in N$; we have

$$
\begin{aligned}
& d_{H}\left(\sum_{i=0}^{k-1} x_{n}\left(\xi_{i}\right)\left(\tau_{i+1}-\tau_{i}\right), E^{n}\right) \\
& \quad \leq \sum_{i=0}^{k-1} d_{H}\left(x_{n}\left(\xi_{i}\right), E^{n}\right)\left(\tau_{i+1}-\tau_{i}\right)
\end{aligned}
$$

for every partition $\left(\tau_{0}, \tau_{1}, \ldots, \tau_{k}\right)$ and every $\left(\xi_{1}, \xi_{2}, \ldots, \xi_{k}\right)$ with $\tau_{i} \leq \xi_{i} \leq \tau_{i+1}$ for $i=0,1,2, \ldots, k-1$. With the continuity of $x_{n}$, we have

$$
d_{H}\left(\int_{a}^{b} x_{n}(\tau) d \tau, E^{n}\right) \leq \int_{a}^{b} d_{H}\left(x_{n}(\tau), E^{n}\right) d \tau .
$$

An application of Fatou's Lemma gives

$$
\varlimsup_{n \rightarrow \infty} d_{H}\left(\int_{a}^{b} x_{n}(\tau) d \tau, E^{n}\right) \leq \int_{a}^{b} \varlimsup_{n \rightarrow \infty} d_{H}\left(x_{n}(\tau), E^{n}\right) d \tau
$$

and with Lebesgue's dominated convergence theorem; since $\varlimsup_{n \rightarrow \infty} d_{H}\left(x_{n}(t), E^{n}\right) \leq \mu(t)$ for $t \in J, m \in N$, we have

$$
\begin{aligned}
\lim _{m \rightarrow \infty} \varlimsup_{n \rightarrow \infty} d_{H}\left(\int_{a}^{b} x_{n}(\tau) d \tau, E^{n}\right) \\
\leq \int_{a}^{b} \lim _{m \rightarrow \infty} \varlimsup_{n \rightarrow \infty} d_{H}\left(x_{n}(\tau), E^{n}\right) d \tau .
\end{aligned}
$$

Therefore, we get

$$
\gamma\left(j \circ\left\{\int_{a}^{b} x_{n}(\tau) d \tau: n \in N\right\}\right) \leq \int_{a}^{b} \phi(\tau) d \tau .
$$

Theorem 13. Let $x_{n}(t):[a, b] \rightarrow E^{n}$ be a sequence of continuously differentiable functions. Assume that there exists $m(t) \in L^{1}[a, b]$ such that

$$
\left\|j \circ x_{n}(t)\right\| \leq m(t), \quad\left\|j \circ x_{n}^{\prime}(t)\right\| \leq m(t)
$$

a.e. $t \in[a, b], n=1,2, \ldots$ Let $h(t)=\gamma\left(j \circ\left\{x_{n}(t): n \in N\right\}\right)$; then $h(t)$ is an absolutely continuous function and $h^{\prime}(t) \leq \gamma(j \circ$ $\left.\left\{x_{n}(t): n \in N\right\}\right)$ a.e. $t \in[a, b]$.

Proof. The absolute continuity of $h$ follows from

$$
\begin{aligned}
\|h(t)-h(s)\| & \leq \gamma\left(\left\{j \circ x_{n}(t)-j \circ x_{n}(s)\right\}\right) \\
& =h\left(j \circ \int_{s}^{t} x_{n}^{\prime}(\tau) d \tau\right) \\
& \leq 2 \cdot \sup _{n \in N}\left\|j \circ \int_{s}^{t} x_{n}^{\prime}(\tau) d \tau\right\| \\
& \leq 2 \cdot \int_{s}^{t} m(\tau) d \tau .
\end{aligned}
$$

We conclude by Theorem 12 that

$$
\begin{gathered}
\gamma\left(j \circ x_{n}(t)\right)-\gamma\left(j \circ x_{n}(t-l)\right) \\
\leq \gamma\left(j \circ \int_{t-l}^{t} x_{n}^{\prime}(\tau) d \tau\right) \\
\leq \int_{t-l}^{t} \gamma\left(j \circ x_{n}^{\prime}(\tau)\right) d \tau .
\end{gathered}
$$


Dividing by $l>0$ and letting $l \rightarrow 0^{+}$give

$$
h^{\prime}(t) \leq \gamma\left(j \circ\left\{x_{n}(t): n \in N\right\}\right) .
$$

Theorem 14. Let $B=\bar{B}\left(x_{0}, r\right)=\left\{x \in E^{n}: D\left(x, x_{0}\right) \leq r\right\}$ and $f:[0, a] \times B \rightarrow E^{n}$ continuous with $D(f(t, x), \widetilde{0}) \leq \mu(t)$ on $[0, a] \times B$ for some $\mu \in L^{1}[0, a]$. Let $b<$ a such that $\int_{0}^{a} \mu(\tau) d \tau<$ $r$ and $J=[0, b]$. One assumes

$$
\begin{array}{r}
\gamma(j \circ f(t, B)) \leq \omega\left(t, \gamma\left(j \circ B^{\prime}\right)\right) \\
\text { for } t \in[0, a], B^{\prime} \subset B,
\end{array}
$$

where $\omega \in U$. Then $(C P)$ has a solution.

Proof. There is a sequence $\left\{x_{n}(t)\right\}$ of approximate solutions to $(C P)$ satisfying

$$
x_{n}^{\prime}(t)=f\left(t, x_{n}(t)\right)+y_{n}(t), \quad x_{n}(t)=x_{0}, y_{n}(t) \leq \frac{1}{n} .
$$

Let $h(t)=\gamma\left(j \circ\left\{x_{n}(t): n \in N\right\}\right)$. We have by Theorem 13 that $h^{\prime}(t) \leq \gamma\left(j \circ\left\{x_{n}(t): n \in N\right\}\right)$ a.e. on $t \in[a, b]$. Moreover, by the properties of $\gamma$, we have

$$
\begin{aligned}
\gamma & \left(j \circ\left\{x_{n}^{\prime}(t)\right\}\right) \\
& \leq \gamma\left(j \circ\left\{f\left(t, x_{n}(t)\right)\right\}\right)+\gamma\left(\left\{j \circ y_{n}(t)\right\}\right) \\
& \leq \omega\left(t, \gamma\left(j \circ\left\{x_{n}(t)\right\}\right)\right) .
\end{aligned}
$$

We claim that $h(t)$ is $o(t)$ for $t \rightarrow 0^{+}$. By the continuity of $f$ and the equicontinuity of the $x_{n}(t)$, we have

$$
\begin{aligned}
x_{n}(t)= & x_{0}+\int_{0}^{t}\left(f\left(\tau, x_{n}(\tau)\right)-f\left(0, x_{0}\right)\right) d \tau \\
& +t f\left(0, x_{0}\right)+\int_{0}^{t} y_{n}(\tau) d \tau \\
= & x_{0}+t f\left(0, x_{0}\right)+z_{n}(t)
\end{aligned}
$$

with $z_{n}(t)=o(t)$ for $t \rightarrow 0^{+}$uniformly in $n$. Since $\omega \in U$, we get $h(t)=0$ on $[a, b]$. Arzala-Ascoli's theorem gives a uniformly convergent subsequence of $x_{n}(t)$ and a standard argument shows that the limit of this subsequence is a solution to $(C P)$ on $[a, b]$.

Next, consider the terminal value problem

$$
x^{\prime}=f(t, x), \quad x(\infty)=x_{0},
$$

where $f:[a, \infty) \times E^{n} \rightarrow E^{n}$ is continuous, $a>0$, and $x_{0} \in$ $E^{n}$. This problem is equivalent to the following initial value problem:

$$
u^{\prime}=-t^{-2} f\left(t^{-1}, u\right), \quad u(0)=x_{0},
$$

by means of the transformation $t \rightarrow t^{-1}$ on $\left(0, a^{-1}\right]$.
Definition 15 (see [23]). A function $\omega:(0, a] \times R^{+} \rightarrow R^{+}$with $\omega \in K\left((0, a] \times R^{+}\right)$is said to be of class $\bar{U}$, denoted by $\omega \in \bar{U}$, if $\phi^{\prime}(t) \leq \omega(t, \phi(t))$ a.e. on $(0, a]$ and $\phi(t)=o(1)$ for $t \rightarrow t^{-1}$ imply $\phi(t)=0$ on $[0, a]$ for each absolutely continuous $\phi$ : $[0, a] \rightarrow R^{+}$.

Theorem 16. Let $E^{n}$ be a fuzzy number space. $B\left(x_{0}, r\right)=\{x \in$ $\left.E^{n}: D\left(x, x_{0}\right) \leq r\right\}$ and $f:[a, \infty) \times B \rightarrow E^{n}$ are continuous. Suppose that $f$ satisfies

(1) $\mu(t)=\sup \left\{D(f, \widetilde{0}): x \in B\left(x_{0}, r\right)\right\}$,

(2) $\gamma(j \circ f(t, B)) \leq \omega(t, \gamma(j \circ B))$ for $t \geq a$,

where $\omega:(0, a] \times R^{+} \rightarrow R^{+}$is such that $t^{-2} \omega\left(t^{-1}, \varrho\right) \in \bar{U}$. Then there exists $b \geq a$ such that the terminal value problem $(C P)^{\prime}$ has a solution in $[b, \infty)$.

Proof. Consider the following problem:

$$
u^{\prime}=-t^{-2} f\left(t^{-1}, u\right), \quad u\left(n^{-1}\right)=x_{0} .
$$

By Theorem 14 and condition (1), the problem (29) has a solution $u_{n}$. Let $u_{n}=x_{0}$ for $t \in\left[0, n^{-1}\right]$. As a consequence of Theorem 13 and the choice of $\omega$, we conclude similar to the proof of Theorem 14 that $(C P)^{\prime}$ has a solution.

\section{On the Asymptotic Equilibrium for Fuzzy Differential Equations}

In this section, we will consider that the system (GCP) has the asymptotic equilibrium. [11]):

Let the functions $[\cdot, \cdot]_{+}$and $[\cdot, \cdot]_{-}$be defined as follows (see

$$
\begin{gathered}
{[u, v]_{+}=\lim _{h \rightarrow 0} \frac{1}{h}(\|j \circ u+h j \circ v\|-\|j \circ u\|),} \\
{[u, v]_{-}=\frac{1}{2}\left([u, v]_{+}-[u,-v]_{+}\right)}
\end{gathered}
$$

for $u, v \in E^{n}$, where $j$ is the embedding operator in Theorem 3.

Theorem 17. For all $u, v, w \in E^{n}$, define $\langle u, v\rangle_{ \pm}=\|j \circ u\|[u, v]_{ \pm}$ and satisfy the following properties:

(1) $\langle u, v\rangle_{ \pm} \leq\|j \circ u\| \cdot\|j \circ v\|$,

(2) $\langle u+v, w\rangle_{-} \leq\langle u, w\rangle_{-}+\|j \circ v\| \cdot\|j w\|$,

(3) if $I$ is an open interval in $R$ and $x: I \rightarrow E^{n}$ is a differential function on $t \in I$, then

$$
\|j \circ x(t)\| \cdot D^{-}\|j \circ x(t)\| \leq\left\langle x^{\prime}(t), x(t)\right\rangle_{-} .
$$

Proof. (1) and (2) are easy consequences of the definition and (3) follows from Lemma 3.6 in [11].

Definition 18. Assume that $\varphi:\left[t^{*}, \infty\right) \rightarrow R$ is a real continuous function, $\varphi\left(t^{*}\right)=0, \varphi(t)>0, t \in\left(t^{*}, \infty\right)$, $\varphi(t)$ is differentiable, and $\varphi^{\prime}(t) \neq 0$ for any $t>t^{*}$. If $\omega(t, u)$ 
is a continuous and $\omega(t, 0) \equiv 0$, the initial value problem $u^{\prime}=\omega(t, u)$ has only solution $u \equiv 0$ and satisfies

$$
\lim _{t \rightarrow t^{*}+0} u(t)=\lim _{t \rightarrow t^{*}+0} \frac{u(t)}{\varphi(t)}=0 .
$$

Then $\omega:\left(t^{*}, \infty\right) \times R^{+} \rightarrow R^{+}$is called a function of $U_{1}$ kind.

Definition 19. Assume that $\omega(t, u)$ is a continuous and $\omega(t, 0) \equiv 0$; the initial value problem $u^{\prime}=\omega(t, u)$ has only solution $u \equiv 0$ and satisfies

$$
\lim _{t \rightarrow t^{*}+0} u(t)=0
$$

for any $t>t^{*}$. Then $\omega:\left(t^{*}, \infty\right) \times R^{+} \rightarrow R^{+}$is called a function of $U_{2}$ kind.

Definition 20 (see [13]). One says that fuzzy equation (CP) has asymptotic equilibrium if every solution of $(C P)$, such that $\left(t_{0}, x_{0}\right) \in R_{+} \times E^{n}$, exists on $\left[t_{0}, \infty\right)$ and tends to a limit $v \in E^{n}$ as $t \rightarrow \infty$, and, conversely, to every given vector $v \in E^{n}$ there exists a solution for fuzzy system $(C P)$ which tends to $v$ as $t \rightarrow \infty$.

Lemma 21 (see [12]). Assume that

(1) $f(t, x)$ is locally Lipschitzian in $x$ for $(t, x) \in J \times E^{n}$,

(2) $D(f(t, x), \widehat{0}) \leq g(t, D(x, \widehat{0}))$, for all $(t, x) \in J \times E^{n}$,

(3) $g \in C[J \times[0, \infty),[0, \infty)], g(t, u)$ is nondecreasing in $u \geq 0$ for each $t \in J$, and maximal solution $r\left(t, t_{0}, u_{0}\right)$ of the scalar initial value problem $u^{\prime}=g(t, u), u\left(t_{0}\right)=u_{0}$ exists throughout $J$.

Then the largest interval of any solution $r\left(t, t_{0}, u_{0}\right)$ of (1) with $D(x, \widehat{0}) \leq u_{0}$ is $J$. In addition, if $r\left(t, t_{0}, u_{0}\right)$ is bounded on $J$, then $\lim _{t \rightarrow \infty} x\left(t, t_{0}, x_{0}\right)$ exists in $\left(E^{n}, D\right)$.

Lemma 22 (see [13]). Under the assumptions of Lemma 21, given $v \in E^{n}$, there exist a $T \in\left[t_{0}, \infty\right)$ and a sequence $\left\{x_{n}(t)\right\}_{n=1}^{\infty}$ defined on $[T, \infty)$, such that

(1) $\left\{x_{n}(t)\right\}_{n=1}^{\infty}$ is equicontinuous on $[T, \infty)$;

(2) $\left\{x_{n}(t)\right\}_{n=1}^{\infty}$ is uniformly bounded on $[T, \infty)$; that is, there exists $M \in R_{+}$, such that $D\left(x_{n}(t), \widehat{0}\right) \leq M$ for all $t \in[T, \infty)$ and for all $n$;

(3) for each $n, x_{n}(t)$ is a solution of $x^{\prime}=f(t, x), x(T+n)=$ $v$.

In the following, we give the main result of this paper.

Theorem 23. Under the assumptions of Lemma 21, $z_{n}(t)=$ $\left\{x_{n}(t), y_{n}(t)\right\}$ is satisfied Lemma 22, and there exists $t^{*} \in$ $\left[T_{0}, \infty\right)$ such that $\left\{z_{n}\left(t^{*}\right)\right\}_{n=1}^{\infty}$ is relatively compact in $E^{n}$ and satisfies the following.

(1) Let $A \subset E^{n}$ is a bounded set; one has $\gamma\left(j \circ f_{1}(t, A)\right) \leq$ $g_{1}\left(t, \gamma\left(j \circ A^{\prime}\right)\right)$ for all $t \in\left[t^{\prime}, \infty\right)$, where $A^{\prime}=\{x$ : there exists $y \in E_{2}^{n}$ such that $\left.(x, y) \in A\right\}$ and $g_{1} \in U_{2} 1$.
(2) For all $\varepsilon>0, t \in R^{+}$, and $r>0$ there exists $\delta=$ $\delta(t, r)>0$ such that $D\left(f_{2}\left(t, x_{1}, y\right), f_{2}\left(t, x_{2}, y\right)\right)<\varepsilon$ with respect to $D(y, \widehat{0}) \leq r$ when $D\left(x_{1}, x_{2}\right)<\delta$; we have $j \circ f_{2}(t, x, y)=h(t)+o\left[\varphi^{\prime}(t)\right]$ when $(t, x, y) \rightarrow$ $\left(t^{*}, x_{0}, y_{0}\right)$, where $h(t)$ is a continuous function and $\varphi(t) \in U_{2}$.

(3) For $\left(t, x, y_{1}\right),\left(t, x, y_{2}\right) \in\left[t^{*}, \infty\right) \times E_{1}^{n} \times E_{2}^{n}$, such that

$$
\begin{gathered}
\left\langle f_{2}\left(t, x, y_{1}\right)-f\left(t, x, y_{2}\right), y_{1}-y_{2}\right\rangle_{-} \\
\leq \omega\left(t, D\left(y_{1}, y_{2}\right)\right) \cdot D\left(y_{1}, y_{2}\right),
\end{gathered}
$$

where $\omega \in U_{1}$ and $\left(x_{0}, y_{0}\right)$ is the limit point of $\left\{x_{n}\left(t^{*}\right), y_{n}\left(t^{*}\right)\right\}$.

Then the fuzzy differential system (GCP) has asymptotic equilibrium.

Proof. Since $z_{n}(t)=\left(x_{n}(t), y_{n}(t)\right)$ is satisfied, $x_{n}^{\prime}=$ $f_{1}\left(t, x_{n}(t), y_{n}(t)\right)$ and $y_{n}^{\prime}=f_{2}\left(t, x_{n}(t), y_{n}(t)\right)$, according to Lemma 10 and Theorem 13, and the following fact: $\left\{x_{n}(t)\right.$ : $\left.n \in N, t \in\left[t^{*}, \infty\right)\right\}$ can produce a subspace of $E_{1}^{n}$; we let $l(t)=\gamma\left(j \circ\left\{x_{n}(t)\right\}\right)$; then we have

$$
\begin{aligned}
l^{\prime}(t) & \leq \gamma\left(j \circ\left\{x_{n}^{\prime}(t): n \in N\right\}\right) \\
& =\gamma\left(j \circ\left\{f_{1}\left(t, x_{n}(t), y_{n}(t)\right)\right\}_{n=1}^{\infty}\right) \\
& \leq g_{1}(t, l(t)) .
\end{aligned}
$$

From the assumption and $l\left(t^{*}\right)=0$, we obtain $l(t)=0$ on $\left[t^{*}, \infty\right)$. Since $\left\{x_{n}(t)\right\}_{n=1}^{\infty}$ is equicontinuous and uniformly bounded, from Ascoli-Arzelar's theorem, then there exists a subsequence of $\left\{x_{n}(t)\right\}_{n=1}^{\infty}$ which uniformly converges to $x(t)$ on any finite closed subset of $\left[t^{*}, \infty\right)$. Without any loss of generality, let $\left\{x_{n}(t)\right\}_{n=1}^{\infty}$ uniformly converge to $x(t)$ on any finite closed subset of $\left[t^{*}, \infty\right)$ and let $\left\{z_{n}\left(t^{*}\right)\right\}_{n=1}^{\infty}$ converge too.

Now, we consider the initial problem

$$
\begin{gathered}
y^{\prime}(t)=f_{2}(t, x(t), y), \\
y\left(t^{*}\right)=y_{0} .
\end{gathered}
$$

Next, we will prove that $\left\{y_{n}(t)\right\}_{n=1}^{\infty}$ converges uniformly to $y(t)$ on any finite closed subset of $\left[t^{*}, \infty\right)$. Let $a_{n m}=$ $D\left(y_{n}\left(t^{*}\right), y_{m}\left(t^{*}\right)\right)=\left\|j \circ y_{n}\left(t^{*}\right)-j \circ y_{m}\left(t^{*}\right)\right\| ;$ then $\lim _{n, m \rightarrow \infty} a_{n m}=0$. And let $\Delta_{n m}(t)=D\left(y_{n}(t), y_{m}(t)\right)=$ $\left\|j \circ y_{n}(t)-j \circ y_{m}(t)\right\|$, according to Theorem 17; we have

$$
\begin{aligned}
& \Delta_{n m} D^{-} \Delta_{n m} \\
& \leq\left\langle y_{n}^{\prime}(t)-y_{m}^{\prime}(t), y_{n}(t)-y_{m}(t)\right\rangle_{-} \\
&=\left\langle f_{2}\left(t, x_{n}(t), y_{n}(t)\right)\right. \\
&\left.\quad-f_{2}\left(t, x_{m}(t), y_{m}(t)\right), y_{n}(t)-y_{m}(t)\right\rangle_{-} \\
& \leq\left\langle f_{2}\left(t, x(t), y_{n}(t)\right)-f_{2}\left(t, x(t), y_{m}(t)\right), y_{n}(t)-y_{m}(t)\right\rangle_{-} \\
&+ {\left[\left\|j \circ f_{2}\left(t, x_{n}(t), y_{m}(t)\right)-j \circ f_{2}\left(t, x(t), y_{n}(t)\right)\right\|\right.} \\
&\left.+\left\|j \circ f_{2}\left(t, x_{m}(t), y_{m}(t)\right)-j \circ f_{2}\left(t, x(t), y_{m}(t)\right)\right\|\right] \Delta_{n m} .
\end{aligned}
$$


Let $\left\{x_{n}(t)\right\}$ converge uniformly to $x(t)$ on $\left[t^{*}, a\right]\left(a>t^{*}\right)$ and combine to condition (2); we have

$$
\begin{aligned}
\Delta_{n m} & D^{-} \Delta_{n m} \\
\leq & \left\langle f_{2}\left(t, x(t), y_{n}(t)\right)\right. \\
& \left.-f_{2}\left(t, x(t), y_{m}(t)\right), y_{n}(t)-y_{m}(t)\right\rangle_{-}+\varepsilon_{n m} \Delta_{n m}
\end{aligned}
$$

where $\lim _{n, m \rightarrow \infty}=0, t \in\left[t^{*}, a\right]$. Then we have

$$
D^{-} \Delta_{n m} \leq \omega\left(t, \Delta_{n m}(t)\right)+\varepsilon_{n m}\left(t \in\left[t^{*}, a\right]\right)
$$

and $\Delta_{n m}\left(t^{*}\right) \rightarrow 0$ when $n, m \rightarrow \infty$. Let $\Delta_{n}=\sup _{m>n} \Delta_{n m}$; then $\lim _{n \rightarrow \infty} \Delta_{n}\left(t^{*}\right)=0$. According to the definition of $\Delta_{n}$, we easily know that $\left\{\Delta_{n}\right\}$ satisfies the following: there exists $M>0$ such that $\left|\Delta_{n}-\Delta_{s}\right| \leq 2 M|s-t|$ and $D^{-} \Delta_{n}(t) \leq$ $\omega\left(t, \Delta_{n}(t)\right)+\varepsilon_{n}\left(s, t \in\left[t^{*}, a\right]\right), \lim _{n \rightarrow \infty} \varepsilon_{n}=0$. From above, we get that $\left\{\Delta_{n}(t)\right\}$ is equicontinuous and uniformly bounded on $\left[t^{*}, a\right]$, so there exists a subsequence converging uniformly to $\Delta(t)$. Since $D^{-} \Delta(t) \leq \omega(t, \Delta(t))$ and $\Delta\left(t^{*}\right)=0$, from the hypotheses of the theorem, fixed $\varepsilon>0$, there exist $t^{\prime} \in$ $\left[t^{*}, a\right], N>0$ such that

$$
D^{-} \Delta_{n m}(t) \leq \varepsilon \varphi^{\prime}(t),
$$

when $n, m>N, t \in\left[t^{*}, t^{\prime}\right]$, which means that

$$
0 \leq \Delta_{n m}(t) \leq \varepsilon \cdot \varphi(t), \quad t \in\left(t^{*}, t^{\prime}\right),
$$

implies

$$
\lim _{t \rightarrow t^{*}} \frac{\Delta(t)}{\varphi(t)}=0
$$

Because $\omega \in U_{1}$, we have $\Delta(t) \equiv 0$. Hence $\left\{\Delta_{n}(t)\right\}$ converge uniformly to $\Delta(t)$; that is, $\left\{\Delta_{n}(t)\right\}$ converge uniformly to 0 . From above, we can get that $\left\{y_{n}(t)\right\}$ converge uniformly to $y(t)$ on $\left[t^{*}, a\right]$. Again, from the arbitrariness of $a$, it is easy to know $z(t)=(x(t), y(t))=\lim _{n \rightarrow \infty}\left(x_{n}(t), y_{n}(t)\right), t \in\left[t^{*}, \infty\right)$ and

$$
\begin{aligned}
& x_{n}(t)=x_{n}\left(t^{*}\right)+\int_{t^{*}}^{t} f_{1}\left(s, x_{n}(s), y_{n}(s)\right) d s, \\
& y_{n}(t)=y_{n}\left(t^{*}\right)+\int_{t^{*}}^{t} f_{2}\left(s, x_{n}(s), y_{n}(s)\right) d s .
\end{aligned}
$$

Since $z_{n}(t)=\left(x_{n}(t), y_{n}(t)\right)$ converge uniformly on a compact set of $\left[t^{*}, \infty\right)$, we have

$$
\begin{gathered}
x(t)=x\left(t^{*}\right)+\int_{t^{*}}^{t} f_{1}(s, x(s), y(s)) d s, \\
y(t)=y\left(t^{*}\right)+\int_{t^{*}}^{t} f_{2}(s, x(s),(s)) d s
\end{gathered}
$$

which means that $z(t)=(x(t), y(t))$ is a solution of fuzzy system $z^{\prime}=f(t, z), z\left(t^{*}\right)=\left(x\left(t^{*}\right), y^{*}(t)\right)$.

Next, we will show $\lim _{t \rightarrow \infty} z(t)=v$. Applying Lemma 22, let $u\left(t, t_{0}, \lambda\right)$ be the largest solution of $u^{\prime}=g(t, u), u\left(t_{0}\right)=\lambda$ for $\lambda \in R^{+}$. According to $g(t, u) \geq 0$, we have $\lim _{t \rightarrow \infty} u(t)=$ $u(\infty)$ and

$$
\lambda=u\left(t_{0}\right) \leq u(t) \leq u(\infty)<\infty .
$$

Since $g(t, u)$ is nondecreasing in $u$ for every $t \in\left[t_{0}, \infty\right)$, we have

$$
\infty>u(\infty)-u\left(t_{0}\right)=\int_{t_{0}}^{\infty} g(s, u(s)) d s \geq \int_{t_{0}}^{\infty} g(s, \lambda) d s
$$

So, there exists an integer $S>t^{*}$ which satisfies

$$
\int_{s}^{\infty} g(t, M) d t \leq \frac{\varepsilon}{2}
$$

where $M=\sup _{n \in N}\left\{D\left(z_{n}(s), \widehat{0}\right), s \in\left[t^{*}, \infty\right)\right\}<\infty$. In addition, there exists $N$; when $n>N$, we have

$$
D\left(z_{n}\left(t^{\prime}\right), z\left(t^{\prime}\right)\right)=\left\|j \circ z_{n}\left(t^{\prime}\right)-j \circ z\left(t^{\prime}\right)\right\|<\frac{\varepsilon}{2} .
$$

Hence,

$$
\begin{aligned}
\left\|j \circ z\left(t^{\prime}\right)-j \circ v\right\| \\
=\left\|j \circ z\left(t^{\prime}\right)-j \circ z_{N+S}(T+N+S)\right\| \\
\leq\left\|j \circ z\left(t^{\prime}\right)-j \circ z_{N+S}\left(t^{\prime}\right)\right\| \\
\quad+\left\|j \circ z_{N+S}\left(t^{\prime}\right)-j \circ z_{N+S}(T+N+S)\right\| \\
\leq \frac{\varepsilon}{2}+D\left(\int_{t^{\prime}}^{T+N+S} f\left(t, z_{N+S}(t) d t\right), \widehat{0}\right) \\
\leq \frac{\varepsilon}{2}+\left|\int_{t^{\prime}}^{T+N+S} g\left(t, D\left(z_{N+S}, \widehat{0}\right)\right) d t\right| \\
\leq \frac{\varepsilon}{2}+\int_{S}^{\infty} g(t, M) d t<\varepsilon .
\end{aligned}
$$

Hence, we have $\lim _{t \rightarrow \infty} z(t)=v$.

When $E^{n}=E_{1}^{n}$ and $f_{2}=0$, we have the following.

Corollary 24. Under the assumptions of Lemma 21, $\left\{x_{n}(t)\right\}_{n=1}^{\infty}$ is satisfied Lemma 22, and there exists $t^{*} \in\left[T_{0}, \infty\right)$ such that $\left\{x_{n}\left(t^{*}\right)\right\}_{n=1}^{\infty}$ is relatively compact in $E^{n}$ and satisfies condition (1) in Theorem 23. Then the fuzzy differential system (CP) has asymptotic equilibrium.

Remark 25. Compared with Theorem 3.4 in [13], the uniformly continuous conditions are removed.

When $E^{n}=E_{2}^{n}$ and $f_{1}=0$, we have the following.

Corollary 26. Under the assumptions of Lemma 21, $\left\{x_{n}(t)\right\}_{n=1}^{\infty}$ is satisfied Lemma 22, and there exists $t^{*} \in\left[T_{0}, \infty\right)$ such that $\left\{x_{n}\left(t^{*}\right)\right\}_{n=1}^{\infty}$ is relatively compact in $E^{n}$ and satisfies

$$
\langle f(t, x)-f(t, y), x-y\rangle_{-} \leq \omega(t, D(x, y)) \cdot D(x, y),
$$

where $t \in[T, \infty), x, y \in E^{n}, \omega \in U_{2}$. Then the fuzzy differential system (CP) has asymptotic equilibrium. 


\section{Conclusion}

In this paper, some global existence and asymptotic equilibrium results for fuzzy differential equations under Bede's derivative for fuzzy-number-valued functions are proved. We apply our main result to the terminal value problem for ordinary differential equations in fuzzy number spaces, a particular situation where we would like to dispense with the uniform continuity assumption of $f$. Our results improve the results given in $[8-10,12,13]$ (where uniform continuity was required), as well as those referred to therein. For future research, we extend the asymptotic stability and global attractivity results for the so-called $(G C P)$ fuzzy systems in our paper.

\section{Conflict of Interests}

The authors declare that there is no conflict of interests regarding the publication of this paper.

\section{Acknowledgments}

The authors are very grateful to the anonymous referees and Professor Junjie Wei for many valuable comments and suggestions which helped in improving the presentation of the paper. The authors would like to thank National Natural Science Foundation of China (no. 11161041) and Fundamental Research Fund for the Central Universities (no. 31920130004 and no. zyz2012079).

\section{References}

[1] Z. Gong and Y. Shao, "Global existence and uniqueness of solutions for fuzzy differential equations under dissipative-type conditions," Computers \& Mathematics with Applications, vol. 56, no. 10, pp. 2716-2723, 2008.

[2] O. Kaleva, "Fuzzy differential equations," Fuzzy Sets and Systems, vol. 24, no. 3, pp. 301-319, 1987.

[3] O. Kaleva, "The Cauchy problem for fuzzy differential equations," Fuzzy Sets and Systems, vol. 35, no. 3, pp. 389-396, 1990.

[4] J. J. Nieto, "The Cauchy problem for continuous fuzzy differential equations," Fuzzy Sets and Systems, vol. 102, no. 2, pp. 259$262,1999$.

[5] M. L. Puri and D. A. Ralescu, "Differentials of fuzzy functions," Journal of Mathematical Analysis and Applications, vol. 91, no. 2, pp. 552-558, 1983.

[6] S. Seikkala, "On the fuzzy initial value problem," Fuzzy Sets and Systems, vol. 24, no. 3, pp. 319-330, 1987.

[7] V. Lakshmikantham and R. N. Mohapatra, Theory of Fuzzy Differential Equations and Inclusions, Taylor \& Francis, London, UK, 2003.

[8] S. Song, C. Wu, and X. Xue, "Existence and uniqueness of Cauchy problem for fuzzy differential equations under dissipative conditions," Computers \& Mathematics with Applications, vol. 51, no. 9-10, pp. 1483-1492, 2006.

[9] C. Wu and S. Song, "Existence theorem to the Cauchy problem of fuzzy differential equations under compactness-type conditions," Information Sciences, vol. 108, pp. 123-134, 1998.

[10] C. Wu, S. Song, and E. S. Lee, "Approximate solutions, existence, and uniqueness of the Cauchy problem of fuzzy differential equations," Journal of Mathematical Analysis and Applications, vol. 202, no. 2, pp. 629-644, 1996.

[11] J. Y. Park and H. K. Han, "Fuzzy differential equations," Fuzzy Sets and Systems, vol. 110, no. 1, pp. 69-77, 2000.

[12] S. Song, L. Guo, and C. Feng, "Global existence of solutions to fuzzy differential equations," Fuzzy Sets and Systems, vol. 115, no. 3, pp. 371-376, 2000.

[13] S. Song, C. Wu, and E. S. Lee, "Asymptotic equilibrium and stability of fuzzy differential equations," Computers \& Mathematics with Applications, vol. 49, no. 7-8, pp. 1267-1277, 2005.

[14] P. Diamond, "Metric spaces of fuzzy sets," Fuzzy Sets and Systems, vol. 100, no. 1, pp. 63-71, 1999.

[15] P. Diamond and P. Kloeden, "Characterization of compact subsets of fuzzy sets," Fuzzy Sets and Systems, vol. 29, no. 3, pp. 341-348, 1989.

[16] C. X. Wu and M. Ma, "Embedding problem of fuzzy number space, part 2," Fuzzy Sets and Systems, vol. 45, no. 2, pp. 189-202, 1992.

[17] B. Zhang, "On measurability of fuzzy-number-valued functions," Fuzzy Sets and Systems, vol. 120, no. 3, pp. 505-509, 2001.

[18] R. J. Aumann, "Integrals of set-valued functions," Journal of Mathematical Analysis and Applications, vol. 12, pp. 1-12, 1965.

[19] B. Bede and S. G. Gal, "Generalizations of the differentiability of fuzzy-number-valued functions with applications to fuzzy differential equations," Fuzzy Sets and Systems, vol. 151, no. 3, pp. 581-599, 2005.

[20] D. Dubois and H. Prade, "Towards fuzzy differential calculus, part 1: integration of fuzzy mappings," Fuzzy Sets and Systems, vol. 8, no. 1, pp. 1-17, 1982.

[21] Y. Chalco-Cano and H. Román-Flores, "On new solutions of fuzzy differential equations," Chaos, Solitons and Fractals, vol. 38, no. 1, pp. 112-119, 2008.

[22] V. Lakshmikantham and S. Leela, Nonlinear Diffrential Equations in Abstract Spaces, Pergamon Press, New York, NY, USA, 1981.

[23] H. Mönch and G.-F. von Harten, "On the Cauchy problem for ordinary differential equations in Banach spaces," Archiv der Mathematik, vol. 39, no. 2, pp. 153-160, 1980. 


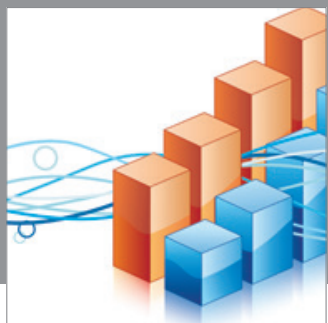

Advances in

Operations Research

mansans

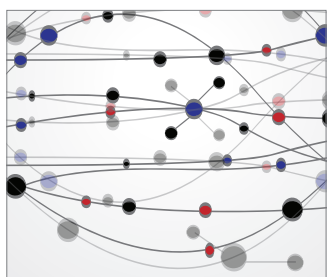

The Scientific World Journal
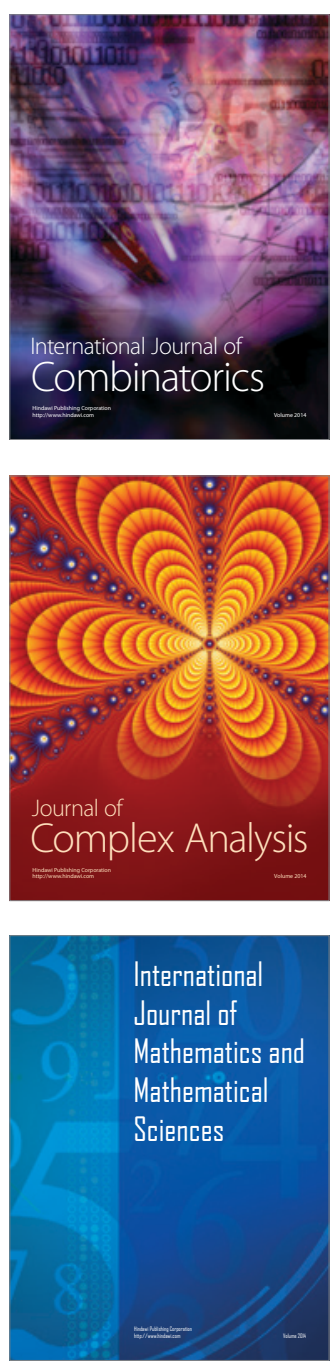
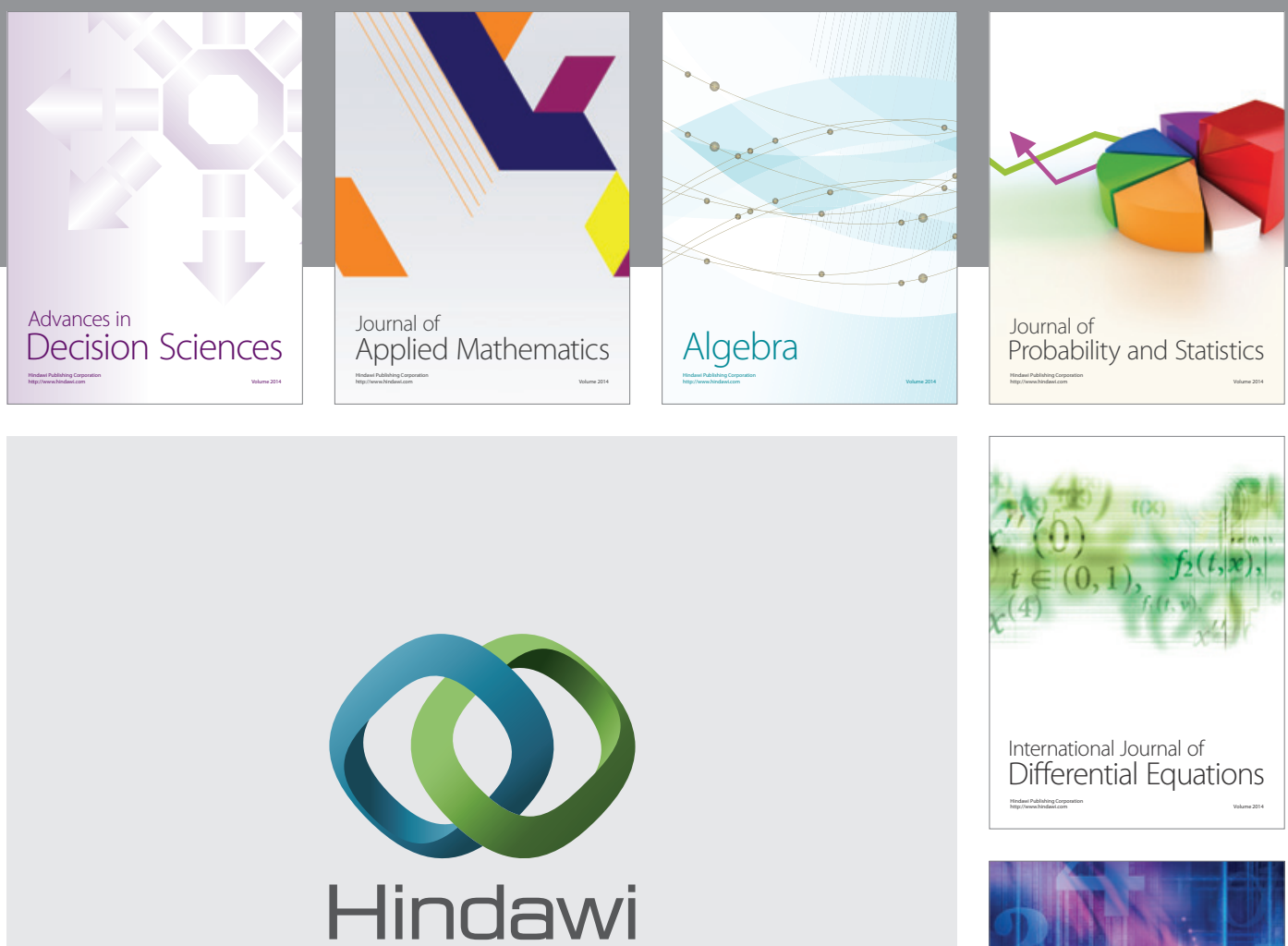

Submit your manuscripts at http://www.hindawi.com
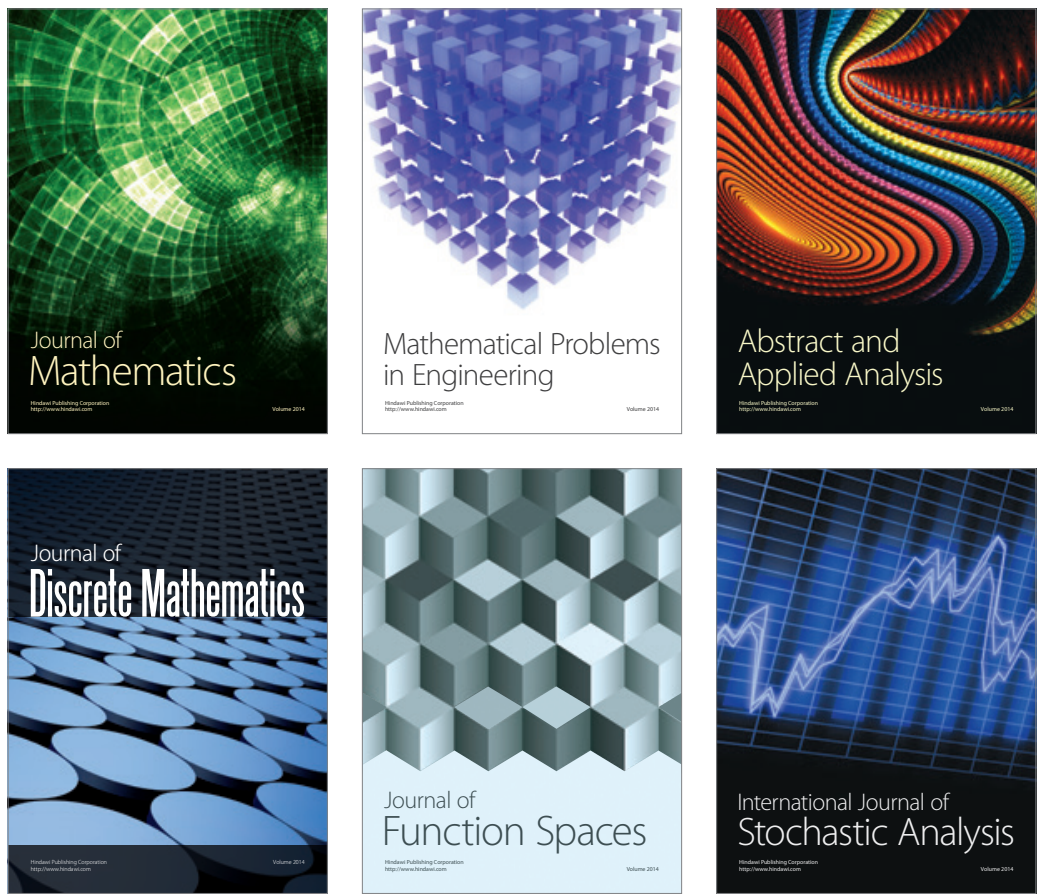

Journal of

Function Spaces

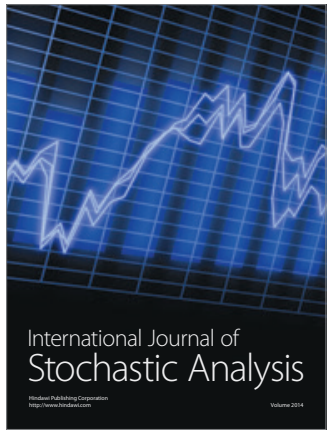

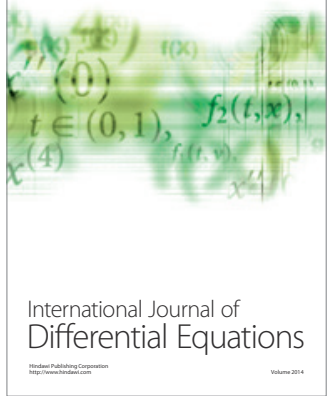
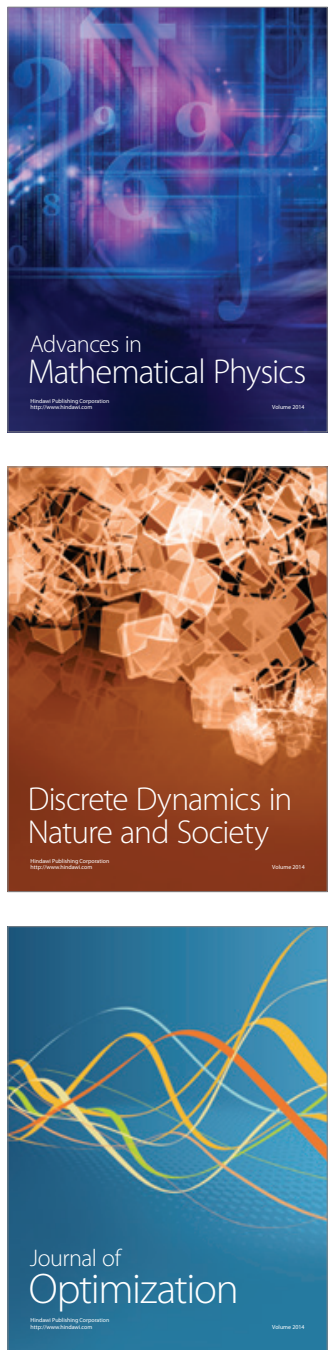